\title{
Perceptions of Rehabilitation Coordinators on Health Information System for Rehabilitation Services in KwaZulu-Natal
}

\author{
Samkelwe Z. Radebe ${ }^{1} \&$ Thembelihle P. Dlungwane ${ }^{1}$ \\ ${ }^{1}$ School of Nursing and Public Health, University of KwaZulu-Natal, Howard College, Durban, South Africa \\ Correspondence: Thembelihle Dlungwane, University of KwaZulu-Natal, School of Nursing and Public Health, \\ Howard College, George Campbell Building $2^{\text {nd }}$ Floor, Durban 4001, South Africa. Tel: 27-31-260-4308. E-mail: \\ dlungwane@ukzn.ac.za
}

Received: November 27, 2019 Accepted: December 15, 2019 Online Published: March 1, 2020

doi:10.5539/gjhs.v12n4p1

URL: https://doi.org/10.5539/gjhs.v12n4p1

\begin{abstract}
Background: A health information system (HIS) is crucial for the planning and management of health services. A HIS provides evidence for programme and policy decisions to ensure better population health outcomes. A HIS aims to improve data collection and handling to extract valuable information thus providing quality health services. Rehabilitation therapists in health facilities in KwaZulu-Natal (KZN) collect data for the monitoring and evaluation of rehabilitation services. Rehabilitation departments in different health facilities have designed data collection tools that are suitable for their institutions, resulting in inconsistency in what is collected across the province of KZN. The study seeks to explore the perceptions of rehabilitation coordinators concerning appropriate indicators for planning and monitoring rehabilitation services in health facilities in KZN.
\end{abstract}

Methods: An exploratory qualitative approach was used. Data was collected through face-to-face in-depth interviews with rehabilitation coordinators who were employed in provincial and district offices in the KZN Department of Health in 2018. Interviews were conducted from June 2018 through September 2018. Each interview was audio-recorded and transcribed verbatim. A thematic analysis was implemented.

Results: The participants highlighted that community outreach, access to multiple assistive devices and discipline specific indicators are appropriate for monitoring rehabilitation services. In addition, integration of rehabilitation indicators with priority programmes such as HIV and TB should be considered.

Conclusion: Rehabilitation coordinators conceptualise the current indicators as limited and insufficient. Rehabilitation indicators should be have linkages with other programmes and reflect the multiple disciplines that fall within rehabilitation services.

Keywords: Health information system, data management for monitoring and evaluation, health information for rehabilitation services

\section{Background}

A health information system (HIS) is crucial for planning and management of responsive health services (National Department of Health, 2013). A HIS aims to improve data collection and handling in order to extract valuable information that can be utilised for health planning, decision-making and resource allocation (AbouZahr \& Boerma, 2005, Hotchkiss, Aqil, Lippeveld, \& Mukooyo, 2010), in order to provide quality health services (Nyamtema, 2010). A HIS provides evidence for programme and policy decisions to ensure better population health outcomes (Nyamtema, 2010).

Many low- and middle-income countries do not have a proper and effective HIS and often cannot afford to invest in data collection and analysis (World Health Organisation, 2017b; Mphatswe et al., 2012). This can result in a gap between policy-makers and health professionals, resulting in a poorly performing health system (AbouZahr \& Boerma, 2005; Mphatswe et al., 2012). Developing countries face a challenge in terms of clinical healthcare workers who are overburdened with work due to staff shortages. Data collection thus becomes 'second best', thereby compromising the quality thereof (Nicol, Bradshaw, Phillips \& Dudley, 2013). Factors influencing HIS performance in developing countries include under-developed HIS infrastructure, inadequate human resource skills, high workloads for healthcare workers and the organisation of HIS (Sibuyi, 2014).

South Africa(SA) has made progress in the establishment and implementation of its HIS since 1994 (English, 
Masilela, Barron, \& Schonfeldt, 2011). There has been a notable improvement in information management, increases in HIS human resources, improvements in the availability of HIS data collection tools, and in the quality of data (Nyamtema, 2010). The quality of data is highly dependent on person skills as well as on the data-collection tools (Kagasi, L. Zungu, \& Hoque, 2013). For data to be useful, it needs to be complete and accurate (Nyamtema, 2010; Mphatswe et al., 2012; Lippeveld, Sauerborn, \& Bodart, 2000). Several organisational factors that contribute to poor quality data have been identified, including lack of staff training, poor supervision and feedback, lack of information use, multiple registers with no standardised tools and insufficient human resources (Hotchkiss et al., 2010; Mate, Bennett, Mphatswe, Barker, \& Rollins, 2009; Mphatswe et al., 2012).

According to the District Health Information System (DHIS) standard operational procedures, healthcare workers are responsible and accountable for ensuring high quality data in individual patient clinical records and on their own data collection and collation tools (NDoH, 2012). However, rehabilitation departments in KwaZulu-Natal (KZN) have no standard tools and guidelines for data collection (Blose, 2017). The national HIS does not adequately integrate rehabilitation-related information $(\mathrm{NDoH}, 2015)$. This hinders evidence-based decision-making, and it is one of the obstacles to strengthening rehabilitation services (WHO, 2017a).

In SA, a national audit on disability research found that there was very little empirical evidence that had evaluated rehabilitation services (Blose, 2017). SA has a quadruple burden of disease with associated impairments that may lead to disability (Sherry, 2014). Medical interventions to save high-risk babies and people living with HIV are improving. However, these patients may be left with impairments that can lead to disability (NDoH, 2015). HIV and TB may cause neurological impairments, dementia, mental illness, TB of the spine, joint disease, pain and fatigue. Research further suggests that many adults with HIV/TB and chronic non-communicable diseases are likely to require rehabilitation and mental health services (Naidoo, Van Wyk, \& Joubert, 2016). Similarly, in terms of maternal and child health, the associated impairments are cerebral palsy, developmental delays, mental illness, and visual and hearing impairments (Sherry, 2014). These patients rely on rehabilitation services to regain maximum function.

Rehabilitation therapists include physiotherapists, occupational therapists, audiologists and speech therapists. All these disciplines have different roles for a patient, but with a similar goal which is to restore and maintain functioning, and prevent disability or loss of function (WHO, 2011). Rehabilitation services need to be evaluated continually for the service to be effective and relevant. However, rehabilitation services have traditionally been neglected in South Africa (Dayal, 2010). Consequently, these services tend to be fragmented and uncoordinated due to the lack of a comprehensive national inter-sectoral rehabilitation policy $(\mathrm{NDoH}, 2015)$

There is currently no standardised health information system in place to support rehabilitation services, and this may influence the allocation of health-related rehabilitation resources to people with disabilities (Stucki, Bickenbach, \& Melvin, 2017). There are also no specific rehabilitation indicators that give a clear picture of what services are rendered daily at facility level (Blose, 2017). The scantiness of appropriate rehabilitation indicators in the national and provincial data sets impacts on the quality of rehabilitation services (NDoH, 2015). Rehabilitation coordinators who are employed at district and provincial level within KZN are responsible for coordinating and overseeing rehabilitation services. In addition, they collate monthly rehabilitation statistics from health facilities. The main aim of this study was to explore perceptions of rehabilitation coordinators regarding appropriate indicators for planning and monitoring rehabilitation services in KZN.

\section{Research Methodology}

\subsection{Research Design}

This study used an exploratory qualitative research design with the purpose of describing the perceptions of rehabilitation coordinators on appropriate rehabilitation indicators for planning and monitoring of rehabilitation services in KZN.

\subsection{Study Setting}

This study was conducted at the KZN Department of Health (KZN DoH) district and provincial offices between June 2018 and September 2018. The study population consisted of rehabilitation coordinators who were employed by KZN DoH at the time of data collection. The participants were interviewed in the boardroom of the district offices where they work. The rehabilitation coordinators that participated in the study were from four districts, and one participant was from the provincial office.

\subsection{Study Population}

A total of five participants who met the inclusion criteria and who participated in the study. In order to gain rich 
information, the study included rehabilitation coordinators that were qualified rehabilitation therapists Rehabilitation coordinators working at KZN DoH district and provincial offices who are qualified rehabilitation therapists (occupational therapist, physiotherapist, speech therapist or audiologist) were invited to participate in the study.

\subsection{Data Collection}

In-depth interviews were conducted with district and provincial rehabilitation coordinators who were recruited through purposive sampling. Purposive sampling is used in qualitative research for the identification and selection of information-rich cases related to the phenomenon of interest ( Patton, 2002). In-depth interviews were chosen in order to gain an in-depth understanding on the perceptions of rehabilitation coordinators concerning appropriate indicators for monitoring rehabilitation services in health facilities in KZN. All interviews were conducted in English.

The interview guide comprised a demographic section as well as questions to guide the interview. The leading question was: "What do you consider as appropriate indicators for rehabilitation services in KZN. This was followed by probing questions to elicit detailed information

\subsection{Data Analysis}

Each interview was audio-recorded and transcribed verbatim. The completeness and quality of the transcript was verified using the observational notes taken during the interviews. The researcher read the written transcript of each participant's interview numerous times in order to gain full understanding of the phenomenon and to then identify codes. A thematic analysis was implemented. Emerging themes were derived through the coding process.

\subsection{Trustworthiness}

Trustworthiness of the findings was achieved through: (i) dependability; (ii) triangulation. Dependability of the data collected was ensured through an audit trail. The main researcher and supervisor served as peer reviewers of the individual in-depth interviews, the level of probing, and the sequence in terms of how the data were collected. Triangulation of data was achieved through the use of individual in-depth interviews and field notes during the interviews.

\subsection{Ethical Considerations}

Ethical approval was granted by the University of KwaZulu-Natal Biomedical Research Ethics Committee (BE075/18) and permission to conduct the study was granted by the Department of Health (HRKM091/18). Participants were given an information letter on the study, and they willingly signed an informed consent. Confidentiality and anonymity of the data were ensured by the researcher.

\section{Results}

The participants consisted of three physiotherapists, one occupational therapist and one speech therapist. Three of participants were females and two were males. The level of management was from district and province. The participant's years of working experience ranged from 10-24 years.

Table 1. Demographic characteristics of participants

\begin{tabular}{|c|c|c|c|c|}
\hline Participant & Professional Background & Level & Gender & Years of working experience \\
\hline AA & Physiotherapist & District & Female & 24 \\
\hline $\mathrm{AB}$ & Physiotherapist & District & Female & 19 \\
\hline $\mathrm{AC}$ & Speech therapist & District & Female & 10 \\
\hline $\mathrm{AD}$ & Occupational therapist & District & Male & 15 \\
\hline $\mathrm{AE}$ & Physiotherapist & Provincial & Male & 19 \\
\hline
\end{tabular}

Significant themes generated from the analysis of participant responses are organized and presented: a)integration of rehabilitation programs with priority programmes; b)rehabilitation discipline-specific indicators; c)access to multiple assistive devices; and d)community outreach indicators (Table 2) 
Table 2. Proposed rehabilitation indicators enaminating from participants' comments

\begin{tabular}{ll}
\hline Rehabilitation indicator & Categories \\
\hline \multirow{3}{*}{ Integrating rehabilitation indicator with priority programmes } & HIV \\
& Under-5s \\
& Over-65s \\
\hline Rehabilitation discipline specific & Audiologist \\
& Physiotherapist \\
& Occupational therapist \\
& Speech therapist \\
& Units, workload \\
& Disease specific \\
\hline Access to multiple assistive devices & Walking aids \\
& Hearing aids \\
& Wheelchairs \\
Community outreach indicators & Number of wheelchairs repaired \\
\hline & Number of old-age homes visited \\
& Number of support groups \\
& Number of campaigns (prevention, in-service) \\
& Number of schools visited \\
& Number of home visits \\
\hline
\end{tabular}

\subsection{Integrating Rehabilitation Indicators With Priority Programmes}

Participants indicated that integrating rehabilitation with priority programmes such as HIV, TB, under-5s, and over-65s will ensure that rehabilitation indicators are standardised across all health facilities. .

'Linking your priority programmes with your rehab patients will help to bring standardisation of rehabilitation indicators. That will mean that rehabilitation patients who have HIV, TB and children with developmental delays are accounted for. So that you could identify [that] HIV and TB programme is taking up to $50 \%$ of our wheelchair budget.' (Participant AA)

'That's why we say it is important that we look at integration; these are the things we need to do. Some of the inputs we can put I think is how is rehabilitation is integrated with the priority programmes. These priority programmes are important according to Department of Health ; so, we need to be looking at how much input we have on that and this will have impact on resource allocation for rehabilitation.' (Participant AB)

There was also an agreement amongst participants that, if rehabilitation services are integrated with the priority programmes, monitoring and evaluation of rehabilitation services in health facilities would improve. Integrating rehabilitation services with priority programmes would ensure that the performance of rehabilitation services is closely monitored. However, integration of rehabilitation indicators into priority programmes would not improve the quality of data collected if training of staff involved in data collection is not implemented.

'[We would take the DHIS data and put that on the spreadsheets with priority programmes. The rehabilitation programmes will be monitored regularly and can easily detect which institutions who have not submitted.' (Participant AA)

'We need to train the staff that is involved in the collection of data so that they understand the importance of data collection and data management. Staff should understand the importance of the data that they are collecting in order to collect good quality in order for the data to inform policy.' (Participant AE)

\subsection{Rehabilitation Discipline-Specific Indicators}

The participants indicated that the current indicators did not reflect the true picture of the rehabilitation disciplines. 
The current head count approach that is used does not reflect on the different disciplines, which fall under rehabilitation.

The participants highlighted that the number of clients accessing rehabilitation services was not specific enough and needed to be broken down to the individual disciplines, which were audiology, physiotherapy, occupational therapy and speech therapy. Participants further proposed that the units of time spent with each patient be captured on the data-collection tools.

'We need a head count of patients seen by speech therapy audiology, physiotherapy and occupational therapy. We really have to have that because how do you assess your services. In a meeting, you would be sitting there saying you have too many rehabilitation clients, but they don't know there is no speech [therapy] in that.' (Participant AA)

'We need to be looking at workload of these therapists. We cannot be capturing patient's head count; it means nothing. If I have four therapists, how much are they capturing, what is their workload. Is it having impact and are we giving quality rehabilitation?' (Participant AB)

'Let everyone show what they are doing, like for speech therapy. Just break it up so that each profession is seen and visible, and I do not know when it goes to the actual work that we do. The indicators also need to show how much time you spend with the patient.' (Participant AC)

Furthermore, a disease- or condition-specific indicator was suggested. These indicators will be discipline specific, with the mention of the conditions to identify which conditions are more prevalent in which areas.

'The current indicator which reports on the head count of patients seen by rehabilitation does not tell me whether I need more for example ore physios or speech therapists. We need to separate the professions, so we know how to boost the numbers because you end up having incomplete rehabilitation team." (Participant AB)

\subsection{Access to Multiple Assistive Devices}

A wide range of assistive devices that are issued by different disciplines within rehabilitation services should be reflected in the indicators. An assistive devices indicator should capture multiple assistive devices and not just focus on wheelchairs, walking aids and hearing aids issued. In addition, participants also mentioned that wheelchair repairs also needed to be included as an indicator.

'.... And even in the assistive devices there should be more than just those on the DHIS; all devices should be counted.' (Participant AC)

'The wheelchair one, I would do it for all the assistive devices. I would not just select wheelchairs; I would take all assistive devices that would be your wheelchairs, your white canes, everything that is done under assistive devices. In addition, your special adapted things that are done for clients, everything that encompasses assistive devices I would put there as one indicator. So that would be issuing and ordering of those assistive devices. I think that would make a lot of sense, it would cover all rehabilitation disciplines.' (Participant AD)

'We do wheelchair repairs, but we are not capturing that on the current indicators.' (Participant AB)

\subsection{Community Outreach Indicator}

Another indicator that came up was the community outreach indicator. The participants indicated the community outreach programmes initiated by therapists are not reflected in the DHIS.

'It [current indicators] doesn't reflect how much work we do behind the scenes, like for instance the impact we have on old-age homes. Do we even capture how many old-age homes we visit and what impact does it have on Department of Social Development? Because that will influence the number of therapists being hired by Department of Social Development.

We also do awareness campaigns with the mothers, the support groups when we look at therapy, OT support groups for children with autism.' There should be indicators that look at the prevention side of rehabilitation. If we pick up problems through early identification, like neonates. For example, number of children under one year that are screened for hearing. We are not looking at the quality of life. (Participant AB)

'The current health reforms are focusing on primary health care re-engineering and community outreach, wardbased outreach teams. Rehabilitation personnel should also be part of these teams hence a community outreach indicator is important for rehabilitation services. (Participant AA)

Participants pointed out data quality issues on the current community outreach indicator as a challenge. They mentioned that data from the clinics is captured on the DHIS by data capturers and sometimes data is incomplete or the data capturers would make up the data. This is further exacerbated by the fact that most are still collecting data 
on paper-based tools; this leads to poor documentation and losing of data.

'...And the data capturers lose the stats and they make up numbers. There are some, in fact majority of time, that is why I do verification because some of the clinics, you go there and there is a gap.' (Participant AB)

'There is a lot of challenges. When I did my verification when I went for my support visit, I found out that there was a lot of gaps and missing data. There would be times where you would find literal gaps, incomplete data.' (Participant AC)

'Most health facilities are using paper based tools and those that have computers have no internet connection. So the data that is collected in those facilities is not well managed the information comes with gaps. '(Participant AD)

\section{Discussion}

The aim of this study was to explore perceptions of rehabilitation coordinators regarding appropriate indicators for planning and monitoring rehabilitation services in KZN. The participants reported that integrating rehabilitation indicators with priority programmes is crucial. This integration will ensure that the rehabilitation services are prioritised, and this could potentially improve the quality of data and resource allocation for rehabilitation services. Special linkages between rehabilitation services and other health programmes should be developed (Sherry, 2014). Priority programmes have shown that they are monitored better locally and globally even though there are still challenges and short comings (Richards et al., 2016).

SA has a quadruple burden of disease with associated impairments that may lead to disability (Sherry, 2014). Medical interventions to save high-risk babies and people with HIV are improving; however, these patients may be left with impairments that can lead to disability (NDoH, 2015). HIV and TB may cause neurological impairments, dementia, mental illness, TB of the spine, joint disease, pain and fatigue. Similarly, in terms of maternal and child health, the associated impairments are cerebral palsy, developmental delays, mental illness, and visual and hearing impairments (Sherry, 2014). These patients rely on rehabilitation services to regain maximum function, indicating that rehabilitation and other programmes are interlinked and need to be integrated. That is why participants suggested HIV, TB, under- $5 \mathrm{~s}$ and over $65 \mathrm{~s}$ be included in the priority programme indicators.

Rehabilitation therapists include physiotherapists, occupational therapists, audiologists and speech therapists. All these disciplines have different roles for a patient, but with a similar goal which is to restore and maintain functioning, and prevent disability or loss of function (WHO, 2011). The participants of our study suggested that there should be discipline-specific indicators (i.e. specific head count for each of the disciplines). These indicators could be further broken down to units to allocate the amount of time spent with each patient and diagnosis. There has been criticism that there are not sufficient to monitor rehabilitation and disability within the HIS and that there were no specific indicators on the type of disability, functioning or access to services (McPherson et al., 2017). Rehabilitation is evaluated by outcomes related to functioning, not by the number of patients seen, and data elements should reflect impairment, levels of function and participation restrictions (McPherson et al., 2017).

Assistive devices provide support for individuals to improve their independence. A variety of assistive devices is provided by different therapists as per the needs of the patient. Participants felt that there should be a list that shows access to multiple assistive devices as indicators for rehabilitation program. The breakdown of assistive devices would facilitate budget allocation, as mentioned by one of the participants. Assistive devices can be divided into: mobility devices, positioning devices, daily living devices, vision devices, hearing devices, communication devices and cognitive devices (WHO, 2010). The provision of appropriate assistive devices is an important part of rehabilitation and is associated with improved function (Visagie, Scheffler, \& Schneider, 2013). However, assistive devices wear out over time and therapists have to do basic repairs on devices like wheelchairs (Visagie et al., 2013).

Patients issued with assistive devices might have barriers that prevent the optimal use of the devices; this might be at home, work, school or in the community (WHO, 2010). The therapist sometimes has to go out to the community to make necessary adaptations to improve the utilisation of assistive devices. This brings about the need to have an indicator for adaptations under the community outreach indicator. The participants felt that they go out to the community and perform the task of adaptions, but these are not reported in the DHIS.

Community outreach interventions form an important part of rehabilitation services and can improve the effectiveness of the programme (WHO, 2011). Outreach aims to bridge the gap by working with the community to determine the needs for assistive devices, offer rehabilitation services and facilitate access to assistive devices (WHO, 2010). Community outreach includes old-age homes, homes, the community setting, the school setting and PHC (clinics and health centres), and it aims to bring services to the people (NDoH, 2015). Rehabilitation services at all levels of care should be reflected in the indicators in order to ensure that appropriate resources are allocated. 
Reliable data is needed to monitor and evaluate community rehabilitation services. However, due to the absence of standardised indicators, such data is rarely available (WHO, 2010).

Participants suggested that community outreach should be included in the rehabilitation data set. This indicator would need to specify the type of outreach, for example, home visits, clinic visits, etc. PHC re-engineering was meant to make healthcare more accessible to people with disabilities, prevent disability through early intervention, and make rehabilitation services available and accessible to all (Sherry, 2014, NDoH, 2015). However, there is a single indicator for rehabilitation at PHC level, which is the provision of assistive devices, and the outcomes are not measured (NDoH, 2015).

The data on rehabilitation services from the health facilities had challenges in terms of incomplete data, incorrect data and late submission. Most participants concluded that it was important to compare source documents for the data captured in the clinics. Having raw data submitted by therapists was pointed out to be of assistance to the district rehabilitation coordinators for verification purposes. In a study done by Sibuyi (2014), the data verification process identified gaps in completeness and consistency, with the data in the DHIS software not corresponding with the data in the source documents.

Once the indicators have been developed, participants suggested training and regular workshops for rehabilitation therapists as ways to improve data quality. A few studies on data quality improvement interventions through training showed improved data quality, particularly increased completeness and accuracy in the health programme data (Mate et al., 2009, Mphatswe et al., 2012, Nyamtema, 2010, Sibuyi, 2014). However, there are studies that have showed that despite training on the DHIS, healthcare workers were not utilising data (Garrib et al., 2008). One of the recommendations from the study conducted by Mate et al. (2009) for improving data quality was for healthcare workers to be trained to perceive data as valuable in making a difference to their performance and in improving service delivery.

Most health facilities in KZN are using paper-based tools and this leads to poor documentation In addition, participants highlighted that lack of IT equipment and internet connectivity is a impedes on the quality data currently collected for rehabilitation services. . Studies have shown the need for a web-based DHIS system because it would be easy to monitor the data remotely as it is captured and also retrieve it quickly when needed (Bhana, 2010, Braa, Heywood \& Sahay, 2012).

\section{1 Study Limitations}

This study focused on rehabilitation coordinators inly and did not include rehabilitation therapists at different levels of care within the health facilities. Further study is needed to explore the perceptions of rehabilitation therapist who are working at different levels of care regarding rehabilitation indicators.

\section{Conclusion}

The results from the analysis reveal that rehabilitation coordinators considered the current indicators as limited and insufficient. Integration of rehabilitation indicators with priority programmes, rehabilitation discipline-specific indicators, access to multiple assistive devices and community outreach indicators must be considered for inclusion in the DHIS for monitoring and planning of rehabilitation services.

\section{Acknowledgements}

The authors acknowledge the participants of the study.

\section{Authors' Contributions}

S.R- Principal author was responsible for the development of the conceptualisation, analysis and writing of the manuscript as part of the Master's degree.

T.D- was responsible for supervising the entire thesis and helped in the writing of the manuscript.

\section{Competing Interests Statement}

The authors declare that they have no competing interests.

\section{References}

AbouZahr, C., \& Boerma, T. ( 2005). Health information systems: The foundations of public health. Bulletin of the World Health Organization, 83(8), 578-583.

Bhana, R. V. (2010). A review of health care indicators in the South African district health information system used for planning, monitoring and evaluation. Retrieved 18 April, 2017, from http://researchspace.ukzn.ac.za 
Blose, S. (2017). Data management workshop for therapists. In Addington hospital.

Braa, J., Heywood, A., \& Sahay, S. (2012). Improving quality and use of data through data-use workshops: Zanzibar, United Republic of Tanzania, Bulletin of the World Health Organization, 90(5), 379-384. https://doi.org/10.2471/BLT.11.099580

Dayal, H. (2010). Provision of rehabilitation services within the District Health System - the experience of rehabilitation managers in facilitating this right for people with disabilities. South African Journal of Occupational Therapy, 40(1), 22-26.

English, R., Masilela, T., Barron, P., \& Schonfeldt, A. (2011). 'Health information systems in South Africa', South African Health Review, 2011(1), 81-89.

Garrib, A., Herbst, K., Dlamini, L., McKenzie, A., Stoops, N., Govender, T., \& Rohde, J. (2008). An evaluation of the district health information system in rural South Africa. South African Medical Journal, 98(7), 549-552.

Hotchkiss, D. R., Aqil, A., Lippeveld, T., \& Mukooyo, E. (2010). Evaluation of the performance of routine information system management (PRISM) framework: Evidence from Uganda. BMC Health Services Research, 10(1), 188. https://doi.org/10.1186/1472-6963-10-188

Kagasi, L.V., Zungu, L., \& Hoque, M. E. ( 2013). Factors influencing the quality of patient data captured by nurses for the tuberculosis control programme in Oshakati District, Namibia. Health SA Gesondheid (Online) 18(1), 1-7. https://doi.org/10.4102/hsag.v18i1.628

Lippeveld, T., Sauerborn, R., \& Bodart, C. (2000). Design and implementation of health information systems. Retrieved 16 May 2017, from http://www.who.int/iris/handle/10665/42289

Mate, K. S., Bennett, B., Mphatswe, W., Barker, P., \& Rollins, N. (2009). Challenges for routine health system data management in a large public programme to prevent mother-to-child HIV transmission in South Africa. PloS One, 4(5), e5483. https://doi.org/10.1371/journal.pone.0005483

McPherson, A., Durham, J., Richards, N., Gouda, H., Rampatige, R., \& Whittaker, M.(2017). Strengthening health information systems for disability-related rehabilitation in LMICs. Health Policy and Planning 32(3), 384-394. https://doi.org/10.1093/heapol/czw140

Mphatswe, W., Mate, K., Bennett, B., Ngidi, H., Reddy, J., Barker, P., \& Rollins, N. (2012). Improving public health information: A data quality intervention in KwaZulu-Natal, South Africa. Bulletin of the World Health Organization, 90(3), 176-182. https://doi.org/10.2471/BLT.11.092759

Naidoo, Deshini, Van Wyk, Jacqueline, \& Joubert, Robin W. E. (2016). Exploring the occupational therapist's role in primary health care: Listening to voices of stakeholders. African Journal of Primary Health Care \& Family Medicine, 8(1), 1-9. https://doi.org/10.4102/phcfm.v8i1.1139

National Department of Health (NDoH). (2012). District Health Information Management System standard operating procedures: Facility level. Retrieved 12 April 2017, from https://www.knowledgehub.org.za/search/elibrary\#kh_modal-node-34634

National Department of Health (NDoH). (2013). District Health Management Information System. Pretoria: National Department of Health.

National Department of Health (NDoH) (2015). Policy framework and strategy for disability and rehabilitation services in South Africa. Retrieved 12 April 2017, from ilifalabantwana.co.za/wp-content/uploads/2016/07/Framework-25-may_1_3.docx

Nicol, E., Bradshaw, D., Phillips, T., \& Dudley, L. (2013). Human factors affecting the quality of routinely collected data in South Africa. Studies in Health Technology and Informatics, 192, 788-792.

Nyamtema, A. S. (2010). Bridging the gaps in the Health Management Information System in the context of a changing health sector. BMC Medical Informatics and Decision Making, 10(1), 36. https://doi.org/10.1186/1472-6947-10-36

Patton. M. Q. (2002). Qualitative research and evaluation methods (3rd ed.). Thousand Oaks, CA: Sage Publications. Qualitative research and evaluation methods (3rd ed.). Thousand Oaks, CA: Sage Publications.

Richards, N. C., Gouda, H. N., Durham, J., Rampatige, R., Rodney, A., \& Whittaker, M. (2016). 2016. Disability, noncommunicable disease and health information. Bulletin of the World Health Organization, 94, 230-2. http://dx.doi.org/10.2471/BLT.15.156869

Sherry, K. (2014). Disability and rehabilitation: Essential considerations for equitable, accessible and 
poverty-reducing health care in South Africa. South African Health Review, 2015, 89-100.

Sibuyi, I. N. (2014). The District Health Information System (DHIS) as a support mechanism for data quality improvement in Waterberg District, Limpopo: An exploration of staff experiences (Unpublished master's dissertation). University of South Africa.

Stucki, G., Bickenbach, J., \& Melvin, J. (2017). Strengthening rehabilitation in health systems worldwide by integrating information on functioning in national health information systems. American Journal of Physical Medicine \& Rehabilitation 96(9), 677-681. https://doi.org/10.1097/PHM.0000000000000688

Visagie, S., Scheffler, E., \& Schneider, M. (2013). Policy implementation in wheelchair service delivery in a rural South African setting. African Journal of Disability, 2(1), a63. https://doi.org/10.4102/ajod.v2i1.63

World Health Organisation (WHO). (2010). Assistive devices, in: Community-based rehabilitation: CBR guidelines. Geneva: World Heath Organisation. Retrieved 25 November 2018, from https://www.ncbi.nlm.nih.gov/books/NBK310951/

World Health Organisation (WHO). (2011). Rehabilitation. World report on disability. Retrieved 25 November 2018, from https://www.who.int/disabilities/world_report/2011/report.pdf

World Health Organisation (WHO). ( 2017a). Rehabilitation 2030: A call for action. Retrieved 13 October 2017, from https://www.who.int/disabilities/care/rehab-2030/en/

World Health Organisation (WHO). (2017b). Rehabilitation in health systems. Retrieved 25 January 2018, from https://www.who.int/disabilities/rehabilitation_health_systems/en/

\section{Copyrights}

Copyright for this article is retained by the author(s), with first publication rights granted to the journal.

This is an open-access article distributed under the terms and conditions of the Creative Commons Attribution license (http://creativecommons.org/licenses/by/4.0/). 